\title{
REDUCED-RANK MMSE DETECTION IN SPACE-TIME CODED SPACE-DIVISION MULTIPLE-ACCESS SYSTEMS
}

\author{
Lie-Liang Yang \\ School of ECS, University of Southampton, SO17 1BJ, UK
}

\section{ABSTRACT}

A multiple-input multiple-output (MIMO) space-division multiple-access (SDMA) system is presented and investigated, when the space-time block coded (STBC) user signals are transmitted over Rayleigh fading channels. In the proposed SDMA system each mobile uses two transmit antennas, while the base-station (BS) receiver employs multiple receive antenna arrays and each receive antenna array consists of several array elements. The MIMO SDMA signals at the BS are detected with the aid of various reduced-rank MMSE schemes. Specifically, three classes of reduced-rank MMSE techniques are investigated in conjunction with the proposed SDMA system. The three classes of reduced-rank MMSE algorithms are derived, respectively, based on the principles of principal components (PC), cross-spectral metric (CSM) and Taylor polynomial approximation (TPA). Our study and simulation results show that the reduced-rank MMSE detection is capable of achieving a satisfactory trade-off between the affordable detection complexity and the achievable detection BER performance.

\section{INTRODUCTION}

In wireless communications MIMO systems equipped with multiple antennas at both the transmitter and receiver hold the promise of attaining substantial spectral efficiency improvements relative to what is achieved today [1]. Recently, MIMO systems have attracted intense research interests in the context of both MIMO theory and applications. It is widely recognized that MIMO systems can be employed for achieving a high capacity [1] and a high diversity order [2], for mitigating the effects of various types of interfering signals [3], and for supporting SDMA. A core principle behind the high spectral efficiency achieved by the MIMO systems is that, when communicating over a rich scattering environment providing nearly independent transmission paths from each transmit antenna to each receive antenna, the multiple antennas employed by the transmitter and/or receiver are capable of providing extra degrees of freedom in the spatial-domain, in addition to the degrees of freedom furnished in the more conventionally exploited timedomain and frequency-domain. This increased degrees of freedom provide novel ideas for increasing the achievable capacity and diversity order of wireless systems, as well as novel ideas for suppressing the effects of intentional or unintentional interfering signals.

In this contribution the application of MIMO principles for supporting SDMA is investigated. Specifically, the synchronous multiple-access communications scenario of Fig. 1 is considered, where each transmitter, such as a mobile user, employs $T_{x}=2$ transmit antennas, while the receiver, which is analogous to the BS, employs $M$ receive antenna arrays. Furthermore, as seen in Fig.1, each of the receive antenna array invokes $L$ array elements or sensors. At the transmitter side Alamouti's STBC scheme based on two transmit antennas is employed for encoding the transmitted symbols, in order to achieve a double-order transmit diversity. By contrast, at the receiver side the signals obtained by sampling the receive antenna arrays' outputs are linearly processed, in order to detect the information transmitted by the different mobile users. Note that, the analysis carried out in our forthcoming discourse can be readily modified to the cases, when the STBC schemes using $T_{x}>2$ transmit antennas are considered.

In this contribution we focus on the reduced-rank MMSE detection of the STBC assisted SDMA signals. Specifically, three classes of reduced-rank MMSE techniques are extended and investigated in conjunction with the proposed SDMA system, so as to achieve low-complexity MMSE detection. The three classes of reduced-rank MMSE algorithms are derived, respectively, based on the principles of PC [4] - [7], CSM [6, 8] and TPA [9]. The characteristics of these reduced-rank MMSE algorithms are considered, and their BER performance is investigated by simulation, when communicating over independent Rayleigh fading channels. Our study and simulation results show that the reduced-rank MMSE detection is capable of achieving a satisfactory trade-off between the affordable detection complexity and the achievable detection BER performance.

\section{REPRESENTATION OF THE RECEIVED SignaL}

Fig. 1 shows the schematic of a SDMA system, where each mobile transmitter employs two transmit antennas, while the base station (BS) receiver has $M$ antenna arrays, each constituted by $L$ array elements. Note that in Fig. 1 the thin lines are related to scalar variables, while the thick lines correspond to vector variables. For the $k$ th user, as shown in Fig. 1, at the transmitter side two adjacent data symbols $x_{1}$ and $x_{2}$ are STBC according to Alamouti's space-time transmission scheme. The resultant STBC symbols are then transmitted in two consecutive symbol periods. In the first symbol period the symbol transmitted from antenna 1 is $x_{k 1}$ and the symbol transmitted from antenna 2 is $x_{k 2}$. By contrast, in the second symbol period the symbol transmitted from antenna 1 is $-x_{k 2}^{*}$ and the symbol transmitted from antenna 2 is $x_{k 1}^{*}$, where the superscript ${ }^{*}$ denotes the complex conjugate.

In our studied BS receiver there are $M$ receiver antenna arrays, as shown in Fig. 1. We assume that the different BS antenna arrays are located sufficiently far apart so that their received signals experience independent fading. As shown in Fig. 1, each of the $M$ antenna arrays consists of $L$ array elements, which are linearly correlated elements separated by a distance of $d$, which is assumed to be half a wavelength in this contribution. We assume that $K$ users are supported by the SDMA system. These users transmit their data synchronously over flat Rayleigh fading channels. Furthermore, we assume 


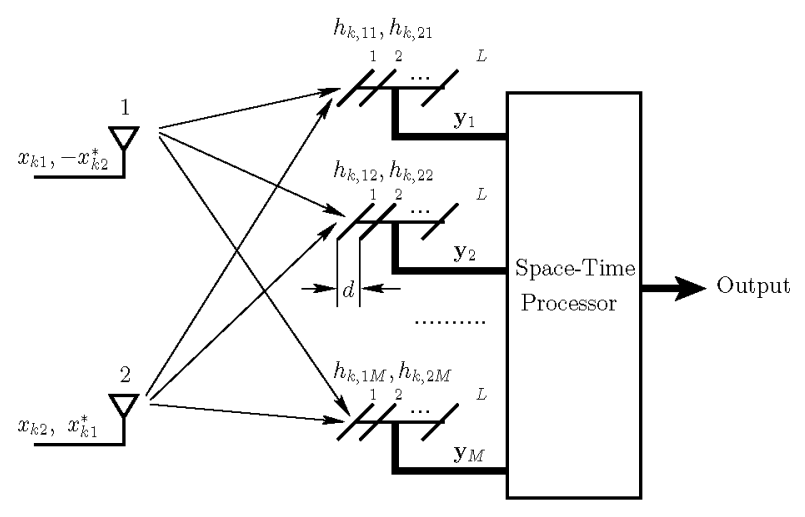

Figure 1: Stylized schematic of the space-time block coding assisted trans mitter, channel model as well as space-time receiver of the SDMA system using two transmit antennas and $M$ receive antenna arrays, each constituted by $L$ elements.

that the complex fading envelope remains constant across two consecutive STBC symbol periods. Consequently, the received signal vector containing the samples collected from $M$ BS antenna arrays in the context of two consecutive symbol periods can be expressed as

$$
\boldsymbol{y}=\sum_{k=1}^{K} \boldsymbol{D}_{k} \boldsymbol{H}_{k} \boldsymbol{x}_{k}+\boldsymbol{n}
$$

where $\boldsymbol{y}$ is a $2 M L$-length vector, $\boldsymbol{n}$ is a $2 M L$-dimensional zeromean AWGN vector, which has a covariance matrix of

$$
\boldsymbol{R}_{n}=E\left[n n^{H}\right]=N_{0} \boldsymbol{I}_{2 M L}
$$

where the superscript ${ }^{H}$ represents the conjugate transpose or Hermitian operation, $N_{0}$ is the complex AWGN's power spectral density, while $\boldsymbol{I}_{2 M L}$ is the identity matrix of size $2 M \mathrm{~L}$. The other arguments in (1) can be expressed as follows.

$$
\begin{aligned}
\boldsymbol{D}_{k} & =\operatorname{diag}\left(\boldsymbol{D}_{k 1}, \boldsymbol{D}_{k 2}, \ldots, \boldsymbol{D}_{k M}\right) \\
\boldsymbol{H}_{k} & =\left[\boldsymbol{H}_{k 1}^{T} \boldsymbol{H}_{k 2}^{T} \ldots \boldsymbol{H}_{k M}^{T}\right]^{T} \\
\boldsymbol{x}_{k} & =\left[x_{k 1} x_{k 2}\right]^{T}
\end{aligned}
$$

where

$\boldsymbol{D}_{k m}=\left[\begin{array}{ll}\boldsymbol{d}_{k m} & \mathbf{0} \\ \mathbf{0} & \boldsymbol{d}_{k m}^{*}\end{array}\right], \boldsymbol{H}_{k m}=\left[\begin{array}{ll}h_{k, 1 m} & h_{k, 2 m} \\ h_{k, 2 m}^{*} & -h_{k, 1 m}^{*}\end{array}\right]$

and $\boldsymbol{d}_{k m}$ denotes the $L$-dimensional complex array vector of the $k$ th user and the $m$ th receive antenna array, which is given by

$$
\boldsymbol{d}_{k m}=\left[1, e^{j \pi \sin \left(\psi_{k m}\right)}, \ldots, e^{j \pi(L-1) \sin \left(\psi_{k m}\right)}\right]^{T}
$$

where $\psi_{k m}$ represents the direction-of-arrival (DOA) corresponding to the $k$ th user and the $m$ th receive antenna array. Furthermore, we assume that $0 \leq \psi_{k m} \leq \pi$. Finally, in (4) $h_{k, i m}=\left|h_{k, i m}\right| e^{j \theta_{k, i m}}$ represents the CIR in the context of the $k$ th user, the $i$ th, $i=1,2$, transmit antenna and the $m$ th, $m=1,2, \ldots, M, \mathrm{BS}$ receive antenna array, where $\left|h_{k, i m}\right|$ represents the fading envelope, obeying the independent Rayleigh distribution.

Based on (1), let us now discuss the detection of the SDMA signals. Specifically, we first derive the full-rank MMSE detector, when assuming that the receive employs the knowledge of $\left\{\boldsymbol{D}_{k}\right\}$ and $\left\{\boldsymbol{H}_{k}\right\}$ associated with all active users. Then, various reduced-rank MMSE detectors are derived.

\section{FULL-RANK MMSE DETECTION}

In the context of the full-rank MMSE detection, the received signal vector $y$ collected at the antenna arrays seen in Fig. 1 is linearly processed by weighting it with the complex weight matrix $W$ of size $2 M L \times 2$, in order to yield the estimate $\hat{x}_{1}$ for the transmitted symbol vector $x_{1}$ as follows:

$$
\hat{\boldsymbol{x}}_{1}=\boldsymbol{W}^{H} \boldsymbol{y}
$$

where the optimum weight matrix $W_{o}$ is chosen such that the mean-square error (MSE) between the transmitted symbol vector $\boldsymbol{x}_{1}$ and the estimated vector $\hat{\boldsymbol{x}}_{1}$ is minimized, yielding [4]

$$
W_{o}=\boldsymbol{R}_{y}^{-1} \boldsymbol{R}_{y x_{1}}
$$

where $R_{y}$ is the auto-correlation matrix of the received signal vector $y$ of (1). When assuming that the received power from all users is the same, $R_{y}$ can be expressed as

$$
\begin{aligned}
\boldsymbol{R}_{y}= & \frac{E_{1}}{2}\left(\boldsymbol{D}_{1} \boldsymbol{H}_{1} \boldsymbol{H}_{1}^{H} \boldsymbol{D}_{1}^{H}\right. \\
& +\underbrace{\left.\sum_{k=2}^{K} \boldsymbol{D}_{k} \boldsymbol{H}_{k} \boldsymbol{H}_{k}^{H} \boldsymbol{D}_{k}^{H}+\frac{2 N_{0}}{E_{1}} \boldsymbol{I}_{2 M L}\right)}_{\Sigma_{2}}
\end{aligned}
$$

where $E_{1}$ represents the transmitted energy of symbol $x_{k 1}$ or symbol $x_{k 2}$, and $\Sigma_{2}$ is the corresponding covariance matrix of the interference plus noise. In (7) $R_{y x_{1}}$ represents the crosscorrelation matrix between the received signal vector $y$ and the desired symbol vector $x_{1}$, which is given by

$$
R_{y x_{1}}=\frac{E_{1}}{2} D_{1} H_{1}
$$

Upon substituting (8) and (9) into (7), the corresponding weight matrix $W_{o}$ is given by

$$
\boldsymbol{W}_{\circ}=\left(\boldsymbol{D}_{1} \boldsymbol{H}_{1} \boldsymbol{H}_{1}^{H} \boldsymbol{D}_{1}^{H}+\boldsymbol{\Sigma}_{2}\right)^{-1} \boldsymbol{D}_{1} \boldsymbol{H}_{1}
$$

Upon invoking the matrix inversion lemma [4], we obtain

$$
W_{o}=\Sigma_{2}^{-1} D_{1} H_{1}\left(H_{1}^{H} D_{1}^{H} \Sigma_{2}^{-1} D_{1} H_{1}+I_{2}\right)^{-1}
$$

Correspondingly, the estimate to $x_{1}$ is given by

$$
\hat{\boldsymbol{x}}_{1}=\left(\boldsymbol{H}_{1}^{H} \boldsymbol{D}_{1}^{H} \boldsymbol{\Sigma}_{2}^{-1} \boldsymbol{D}_{1} \boldsymbol{H}_{1}+\boldsymbol{I}_{2}\right)^{-1} \boldsymbol{H}_{1}^{H} \boldsymbol{D}_{1}^{H} \boldsymbol{\Sigma}_{2}^{-1} \boldsymbol{y}
$$

Furthermore, it can be shown that the achievable MMSE can be expressed as

$$
\operatorname{MMSE}=1-\frac{1}{E_{1}} \operatorname{Trace}\left(\boldsymbol{R}_{y x_{1}}^{H} \boldsymbol{R}_{y}^{-1} \boldsymbol{R}_{y x_{1}}\right)
$$


where $\operatorname{Trace}(\boldsymbol{A})$ represents the trace of $\boldsymbol{A}$.

Above we have considered the full-rank MMSE detection of the SDMA signals. As seen in (12), the detection complexity is dominated by inverting the matrix $\Sigma_{2}$ of rank $2 M L$, where $M$ is the number of receive antenna arrays, and $L$ is the number of array elements per receive antenna array. Hence, the rank of $2 M L$ might be very high, when a SDMA system employs a high number of antenna arrays and/or each of them has a high number of sensors, for example, in order to support a high number of users. Consequently, the complexity of the MMSE multiuser detectors might be extreme. Below we investigate a variety of reduced-rank MMSE detectors, which invoke the inversion of matrices having the rank that may be significantly lower than $2 M L$.

\section{REDUCED-RANK MMSE DETECTION}

A reduced-rank MMSE detector reduces the number of coefficients to be estimated or optimized by projecting the received signal vector onto a lower dimensional subspace [4]. Specifically, let $\boldsymbol{P}_{U}$ be the $(2 M L \times U)$ processing matrix with its column vectors forming a basis of a $U$-dimensional subspace, where $U<2 M L$. Then, for a given received $2 M L$ dimensional vector $y$, the $U$-dimensional vector in the subspace can be expressed as

$$
\overline{\boldsymbol{y}}=\underbrace{\left(\boldsymbol{P}_{U}^{H} \boldsymbol{P}_{U}\right)^{-1} \boldsymbol{P}_{U}^{H}}_{\boldsymbol{S}_{U}^{H}} y
$$

where $\left(\boldsymbol{P}_{U}^{H} \boldsymbol{P}_{U}\right)^{-1}$ represents a normalization factor. Note that, an "overbar" is used for indicating the operation in the reduced-rank subspace.

In the subspace, $\bar{y}$ is the vector input to the MMSE detector, where it is processed by the MMSE filter and output estimates for the transmitted space-time symbols $\left\{\boldsymbol{x}_{1}\right\}$. Upon following the principles of MMSE [4], it can be shown that we have

$$
\hat{\boldsymbol{x}}_{1}=\overline{\boldsymbol{W}}_{o}^{H} \overline{\boldsymbol{y}}
$$

where the weight matrix is now expressed as

$$
\overline{\boldsymbol{W}}_{o}=\frac{E_{1}}{2}\left(\boldsymbol{S}_{U}^{H} \boldsymbol{R}_{y} \boldsymbol{S}_{U}\right)^{-1} \boldsymbol{S}_{U}^{H} \boldsymbol{D}_{1} \boldsymbol{H}_{1}
$$

According to the above analysis, we can know that, for reduced-rank MMSE detection, our main task is to derive a $U$ dimensional detection subspace $P_{U}$, so that it can approximate the original $2 M L$-dimensional space as closely as possible or that the resulted MMSE is as low as possible. Below a range of rank-reduction algorithms are extended and investigated associated with our SDMA systems. Note that, in our forthcoming discussion, for simplicity, we ignore the factor of $E_{1} / 2$, when invoking the autocorrelation matrix $\boldsymbol{R}_{y}$ or the cross-correlation matrix $\boldsymbol{R}_{y x_{1}}$, since it is either removed during the derivations or does not affect the final results at all. This can be shown by (16), where the factor of $E_{1} / 2$ will be removed, since the same factor is also contained in $R_{y}$ as seen in (8).

\section{A. Eigenspace: Principal Components}

The principal components (PC) based rank-reduction, which is based on the eigen-decomposition of the auto-correlation ma- trix, has received wide research and application [4]. Specifically, the PC-based rank-reduction starts with expressing the Hermitian auto-correlation matrix $\boldsymbol{R}_{y}$, as shown in (8), using its eigen-decomposition as

$$
\boldsymbol{R}_{y}=\boldsymbol{\Phi} \Lambda \boldsymbol{\Phi}^{H}
$$

where $\Phi$ is the orthonormal matrix whose columns consist of the eigenvectors of $R_{y}$, i.e., $\Phi$ is in the form of

$$
\boldsymbol{\Phi}=\left[\boldsymbol{\phi}_{1}, \boldsymbol{\phi}_{2}, \ldots, \boldsymbol{\phi}_{2 M L}\right]
$$

where $\phi_{i}$ is the eigenvector corresponding to the eigenvalue of $\lambda_{i}$. In (17) $\Lambda$ is the diagonal matrix, which is given by

$$
\boldsymbol{\Lambda}=\operatorname{diag}\left\{\lambda_{1}, \lambda_{2}, \ldots, \lambda_{2 M L}\right\}
$$

Let us assume that the eigenvalues are ordered as $\lambda_{1} \geq \lambda_{2} \geq$ $\ldots \geq \lambda_{2 M L}$. Then, for a given subspace of dimension $U$, the processing matrix $\boldsymbol{P}_{U}$ in (14) in the context of PC is constituted by the first $U$ columns of $\Phi$, i.e., $\boldsymbol{P}_{U}=\left[\boldsymbol{\phi}_{1}, \boldsymbol{\phi}_{2}, \ldots, \boldsymbol{\phi}_{U}\right]$.

\section{B. Eigenspace: Cross-Spectral Metric}

The cross-spectral metric (CSM) based technique for reducedrank filtering has been investigated in [4] - [7]. This CSM technique is designed with attempting to minimize the achievable MMSE of the reduced-rank MMSE detector. Specifically, with the aid of (17), (18) and (19), we have [4]

$$
\boldsymbol{R}_{y}=\sum_{i=1}^{2 M L} \lambda_{i} \phi_{i} \phi_{i}^{H}, \quad \boldsymbol{R}_{y}^{-1}=\sum_{i=1}^{2 M L} \frac{\boldsymbol{\phi}_{i} \boldsymbol{\phi}_{i}^{H}}{\lambda_{i}}
$$

Upon substituting $R_{y}^{-1}$ into (13) and remembering ignoring the factor $E_{1} / 2$, the MMSE of the full-rank MMSE detector can be written as

$$
\operatorname{MMSE}=1-\frac{1}{2} \operatorname{Trace}\left(\sum_{i=1}^{2 M L} \frac{\left\|\boldsymbol{H}_{1}^{H} D_{1}^{H} \boldsymbol{\phi}_{i}\right\|^{2}}{\lambda_{i}}\right)
$$

Based on (21), for the CSM-based reduced-rank detection, the subspace $\boldsymbol{P}_{U}$ is constituted by the $U$ eigenvectors of $\boldsymbol{R}_{y}$, which correspond with the largest $U$ values of the CSMs defined as

$$
\frac{\left\|H_{1}^{H} D_{1}^{H} \phi_{i}\right\|^{2}}{\lambda_{i}}, i=1, \ldots, 2 M L
$$

The principles behind the CSM-based reduced-rank technique can be readily understood by referring to (21), which shows that, for a given value of $U$, the $U$ terms having the highest CSMs in the form of (22) must be maintained, in order for achieving the minimum MSE. Hence, the detection subspace must correspondingly be constituted by the $U$ eigenvectors achieving these $U$ highest CSMs.

\section{Taylor Polynomial Approximation}

The reduced-rank processing matrix $\boldsymbol{P}_{U}$ based on Taylor polynomial approximation (TPA) is derived by first Taylor expanding the matrix $R_{y}^{-1}$ in (7), which can be described as follows. 
Let $\lambda_{\max }$ be the maximum eigenvalue of $R_{y}$. Let $\rho$ be a constant satisfying $0<\rho<1 / \lambda_{\max }$. Then, the matrix $\boldsymbol{R}_{y}^{-1}$ can be Taylor expanded as

$$
\begin{aligned}
\boldsymbol{R}_{y}^{-1}=\rho\left(\rho \boldsymbol{R}_{y}\right)^{-1} & =\rho\left[\boldsymbol{I}-\left(\boldsymbol{I}-\rho \boldsymbol{R}_{y}\right)\right]^{-1} \\
& =\rho \sum_{n=0}^{\infty}\left(\boldsymbol{I}-\rho \boldsymbol{R}_{y}\right)^{n}
\end{aligned}
$$

Let the first $U / 2$ terms in (23) be used in order to approximate $R_{y}^{-1}$. Then we have

$$
\begin{aligned}
\boldsymbol{R}_{y}^{-1} & \approx \rho \sum_{n=0}^{U / 2-1}\left(\boldsymbol{I}-\rho \boldsymbol{R}_{y}\right)^{n} \\
& =a_{0} \boldsymbol{I}+a_{1} \boldsymbol{R}_{y}+\ldots+a_{U / 2-1} \boldsymbol{R}_{y}^{U / 2-1}
\end{aligned}
$$

where the coefficients $\left\{a_{n}\right\}$ are determined by $\rho$ associated with the expansion of (24). Consequently, the estimate to $\boldsymbol{x}_{1}$ can be approximately expressed as

$$
\hat{\boldsymbol{x}}_{1} \approx\left(a_{0} \boldsymbol{D}_{1} \boldsymbol{H}_{1}+\ldots+a_{U / 2-1} \boldsymbol{R}_{y}^{U / 2-1} \boldsymbol{D}_{1} \boldsymbol{H}_{1}\right)^{H} \boldsymbol{y}
$$

However, determining the coefficients $\left\{a_{n}\right\}$ in (26) may result in high complexity. Furthermore, the finite order approximations that result from tail-cutting of infinite order approximations generally do not lead to the best fit among all approximations of the same order. Hence, for the purpose of reducedrank MMSE detection, we can design the processing matrix $P_{U}$ as

$$
\boldsymbol{P}_{U}=\left[\boldsymbol{D}_{1} \boldsymbol{H}_{1}, \boldsymbol{R}_{y} \boldsymbol{D}_{1} \boldsymbol{H}_{1}, \ldots, \boldsymbol{R}_{y}^{U / 2-1} \boldsymbol{D}_{1} \boldsymbol{H}_{1}\right]
$$

which is a $(2 M L \times U)$-dimensional matrix. Then, the received space-time vector $y$ is projected onto the subspace determined by the columns of $P_{U}$, and is processed by an optimum weight matrix $\bar{W}_{o}$ derived in MMSE sense.

The TPA technique has been originally applied for deriving the reduced-rank linear detectors for CDMA systems [9]. In comparison with the other reduced-rank MMSE detectors discussed previously in this section, the TPA assisted reduced-rank MMSE detection does not depend on the eigendecomposition of the auto-correlation matrix $\boldsymbol{R}_{y}$. According to [5] and our simulation results in Section V., the performance of this type of reduced-rank MMSE detector is capable of converging to full-rank performance with a rank of $U$ significantly lower than that of the other types of reduced-rank MMSE detectors considered in this contribution. Furthermore, the study in [5] shows that the rank $U$ needed to achieve full-rank performance does not scale with the system size determined by the number of users, $K$, and the antenna array resultant dimensions, $2 M L$. Hence, the rank $U$ of the detection subspace can usually be significantly lower than the number of users supported by the SDMA system, when the number of users supported is high. Let us now provide a range of simulation results.

\section{EXAMPLES OF PERFORMANCE RESULTS}

In this section we provide a range of simulation results for illustrating and comparing the achievable performance of the proposed SDMA system in conjunction with the various detection schemes considered in this contribution. In our simulations binary phase shift keying (BPSK) baseband modulation was assumed.

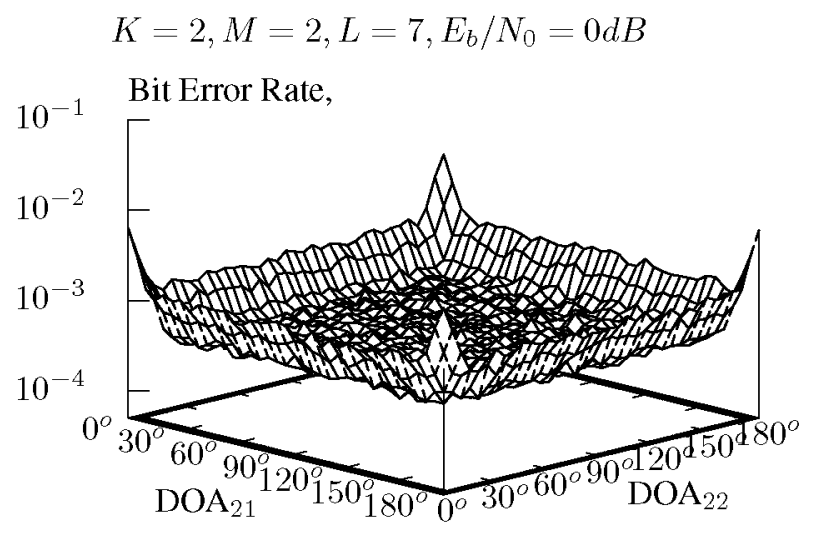

Figure 2: BER versus DOAs performance of the SDMA system using $T x=$ 2 transmit antennas, $M=2$ receive antenna arrays of each having $L=7$ sensors, and supporting $K=2$ users, when communicating over Rayleigh fading channels and when using the full-rank MMSE multiuser detection.

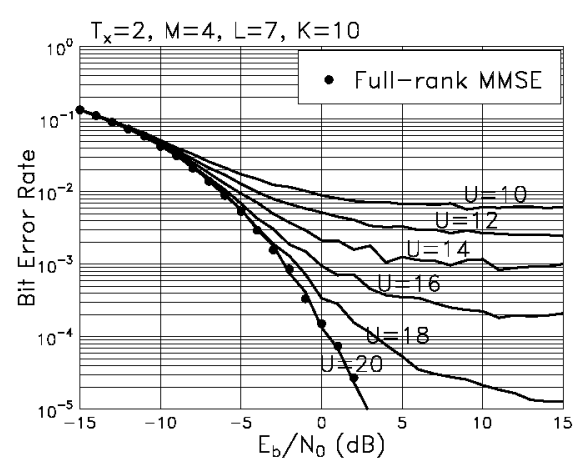

Figure 3: Principal components: BER versus SNR per bit performance of the SDMA system using $T x=2$ transmit antennas, $M=4$ receive antenna arrays of each having $L=7$ sensors, and supporting $K=10$ users, when communicating over Rayleigh fading channels.

In order to gain an insight into the effect of the DOAs on the BER performance, Fig. 2 shows the BER versus the DOA angles performance for the SDMA systems, when the full-rank MMSE multiuser detector of (12) employed the knowledge of $\left\{\boldsymbol{H}_{k}\right\}_{k=1}^{2}$ and $\left\{\boldsymbol{D}_{k}\right\}_{k=1}^{2}$. In Fig. $2 \mathrm{DOA}_{21}=\psi_{21}-\psi_{11}$ and DOA $_{22}=\psi_{22}-\psi_{12}$, which represent the DOA differences between user 2's and user 1's signals received, respectively, by the first and second antenna arrays. Without loss of generality, we assumed that $\psi_{11}=\psi_{12}=0^{\circ}$, while that $\psi_{21}$ and $\psi_{22}$ were assumed independent and took values in the range of $\left[0^{\circ}, 180^{\circ}\right)$. Furthermore, in our simulations the SNR per bit of $E_{b} / N_{0}$ was assumed to be $0 \mathrm{~dB}$. From the results of Fig. 2, we observe that, when the interfering signals spatially arrive 
from the DOAs different from that of the desired signals, the multiuser interference may be substantially mitigated by the receiver with the aid of the beamforming and MMSE processing. When the DOAs of both the interfering user and the reference user are nearly identical, the BER achieved is higher than that achieved, when the interfering signals spatially arrive from different DOAs of the desired signals.

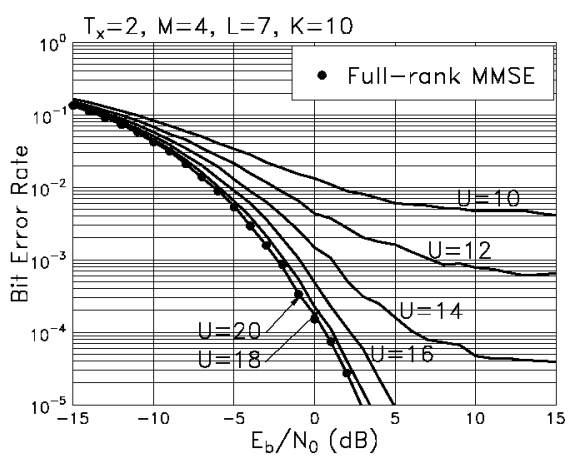

Figure 4: Cross-spectral metric: BER versus SNR per bit performance of the SDMA system using $T x=2$ transmit antennas, $M=4$ receive antenna arrays of each having $L=7$ sensors, and supporting $K=10$ users, when communicating over Rayleigh fading channels.

Figs. 3 - 5 show the BER performance of the SDMA system, when using various reduced-rank MMSE detectors. From the results seen in Figs. 3 - 5, we can have the following observations.

- The BER performance improves, when increasing the rank, $U$, of the detection subspace, provided that the rank $U$ is lower than the rank of the signal subspace, or in Fig.5 is lower than 14 . When the rank, $U$, of the detection subspace reaches the rank 20 of the signal subspace, all the reduced-rank MMSE detectors are capable of achieving the BER performance of a corresponding full-rank MMSE detector.

- As shown in Figs. 3 and 4, for those reduced-rank MMSE detectors designed based on PC (Fig. 3) and CSM (Fig. 4), when the rank, $U$, of the detection subspace is lower than the rank of the signal subspace, BER error floors may be observed. Typically, a lower rank of the detection subspace results in a higher BER floor. Hence, we are implied that these types of reduced-rank MMSE detectors need to acquire the knowledge about the rank of the signal subspace. Otherwise, they may experience near-far problem.

- It can be observed that, for any a given rank $U$ of the detection subspace, the CSM-based reduced-rank MMSE detector outperforms the PC-based reduced-rank detector. Furthermore, as shown in Fig. 4, no BER floors are observed for the cases of $U=16$ and 18 within the BER range considered, even the rank $U$ of the detection subspace is lower than the rank 20 of the signal subspace.

- Finally, it can be shown that the TPA-based reduced-rank MMSE detector outperforms all the other reduced-rank detectors and achieves the best BER performance for a given value of $U$. From the results of Fig. 5 we can see that the full-rank performance can be achieved, provided that the rank of the detection subspace is not lower than 14 , which is significantly lower than 20 of the rank of the signal subspace. Furthermore, as we mentioned previously in Section C., the TPA scheme does not invoke complex eigen-decomposition for forming the detection subspace.

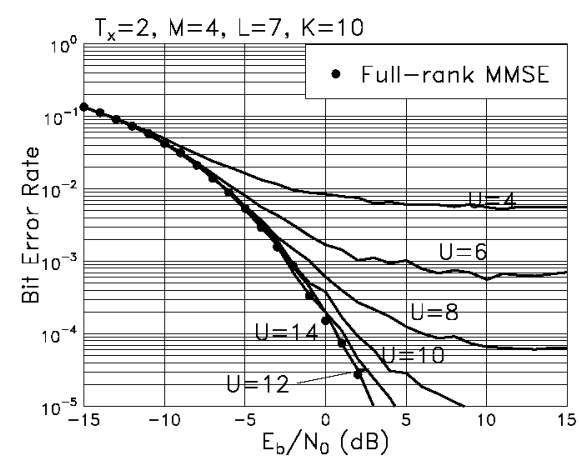

Figure 5: Taylor polynomial approximation: BER versus SNR per bit performance of the SDMA system using $T x=2$ transmit antennas, $M=4$ receive antenna arrays of each having $L=7$ sensors, and supporting $K=10$ users, when communicating over Rayleigh fading channels.

\section{ACKNOWLEDGMENT}

The author would like to acknowledge with thanks the financial assistance from EPSRC of UK.

\section{REFERENCES}

[1] I. E. Telatar, "Capacity of multiantenna Gaussian channels," European Trans. on Telecomm., vol. 10, pp. 585-595, Nov./Dec. 1999.

[2] V. Tarokh, N. Seshadri, and A. R. Calderbank, "Space-time codes for high data rate wireless communication: performance criterion and code construction," IEEE Trans. on Inf. Theory, vol. 44, pp. 744-765, Mar. 1998.

[3] J. H. Winters, "Optimum combining in digital mobile radio with cochannel interference," IEEE J. on Select. Areas in Comm., vol. SAC-2, pp. 528-539, July 1984.

[4] H. L. V. Trees, Optimum Array Processing. Wiley Interscience, 2002.

[5] J. S. Goldstein, I. S. Reed, and L. L. Scharf, "A multistage representation of the Wiener filter based on orthogonal projections," IEEE Trans. on Inf. Theory, vol. 44, pp. 1943 - 2959, Nov. 1998.

[6] J. S. Goldstein and I. S. Reed, "Subspace selection for partially adaptive sensor array processing," IEEE Trans. on Aerospace and Elect. Systems, vol. 33, pp. 539 - 544, Apr. 1997.

[7] M. L. Honig and J. S. Goldstein, "Adaptive reduced-rank interference suppression based on the multistage Wiener filter," IEEE Trans. on Comm., vol. 50, pp. 986 - 994, June 2002.

[8] J. S. Goldstein and I. S. Reed, "Reduced-rank adaptive filtering," IEEE Trans. on Signal Proc., vol. 45, pp, 492 - 496, Feb. 1997.

[9] S. Moshavi, E. G. Kanterakis, and D. L. Schilling, "Multistage linear receivers for DS-CDMA systems," Int. J. of Wireless Inf. Networks, vol. 3 , pp. $1-17$, Jan. 1996. 